\title{
Monthly intravesical bacillus Calmette-Guérin maintenance therapy for non-muscle-invasive bladder cancer: 10-year experience in a single institute
}

\author{
KOO HAN YOO, TAE JOON LIM and SUNG-GOO CHANG \\ Department of Urology, School of Medicine, Kyung Hee University, Seoul, Republic of Korea
}

Received October 7, 2011; Accepted November 28, 2011

DOI: $10.3892 /$ etm.2011.400

\begin{abstract}
Intravesical bacillus Calmette-Guérin (BCG) therapy is the standard prophylaxis for recurrence of non-muscle-invasive bladder cancer (NMIBC). The aim of this study was to confirm the recurrence- and progressionpreventing efficacy and safety of 12 times monthly BCG maintenance therapy for NMIBC. This study included 126 patients diagnosed with Ta, T1 and carcinoma in situ bladder cancer between January 2000 and December 2009. Thirty-four patients in the no maintenance group received a single 6-week course of intravesical immunotherapy after transurethral resection of the bladder tumor (TUR-BT). Ninety-two patients in the maintenance group received a 12-month course after a single 6-week course of intravesical immunotherapy. Recurrence, progression and side effects were assessed. End-points were recurrence-free survival (RFS), progression-free survival (PFS) and disease-specific survival (DSS). The estimated median RFS was 87 months (95\% CI 53.0-120.9) in the maintenance group and 48 months (95\% CI 0-96.8) in the no maintenance group (log-rank test; $\mathrm{P}=0.002)$. The 2-year cumulative RFS rates were $77.3 \%$ in the maintenance group and $52.6 \%$ in the no maintenance group. Median PFS and DSS were not estimable in both groups. The 2-year cumulative PFS rates were $91.1 \%$ in the maintenance group and $80.5 \%$ in the no maintenance group (log-rank test; $\mathrm{P}=0.178$ ). The 2 -year cumulative DSS rates were $97.7 \%$ in the maintenance group and $91.4 \%$ in the no maintenance group (log-rank test; $\mathrm{P}=0.111$ ). The overall side effects were $40.2 \%$ in the maintenance group and $44.1 \%$ in the no maintenance group. Monthly maintenance immunotherapy appears to increase RFS rates in high-risk NMIBC. Further study is required to evaluate the efficacy of BCG monthly maintenence for increasing the PFS rate.
\end{abstract}

Correspondence to: Professor Sung-Goo Chang, Department of Urology, Kyung Hee University Hospital, \#1 Hoegi-dong, Dongdaemun-ku, Seoul 130-702, Republic of Korea

E-mail: sgchang@khu.ac.kr

Key words: bladder cancer, intravesical therapy, bacillus CalmetteGuérin, maintenance instillation

\section{Introduction}

In 2008, an estimated 386,300 new cases and 150,200 deaths from bladder cancer were reported. The majority of bladder cancer occurs in males and there is a 14-fold variation in incidence internationally (1). Non-muscle-invasive bladder cancer (NMIBC) accounts for approximately $80 \%$ of total bladder cancer cases (2). Intravesical bacillus Calmette-Guérin (BCG) therapy is the standard prophylaxis for recurrence of NMIBC (3). However, after a single 6-week course of intravesical immunotherapy after transurethral resection of bladder tumor (TUR-BT), the 5-year recurrence rates range from 16 to $59 \%$ (4-6).

A randomized Southwest Oncology Group (SWOG) study reported the results of intravesical maintenance immunotherapy. This revealed that the median recurrence-free survival (RFS) time was twice as long in the 3-week maintenance arm compared to the no maintenance arm, and patients had significantly longer worsening-free survival (4). At least 1 week following biopsy of carcinoma in situ (CIS) and resection of any stage Ta or T1 transitional cell carcinomas, patients were started on a 6-week induction course of intravesical and percutaneous Connaught BCG. Maintenance therapy of the SWOG study consisted of intravesical and percutaneous BCG each week for 3 weeks, administered 3, 6, 12, 18, 24, 30 and 36 months from the initiation of the induction therapy.

A number of randomized studies have focused on the benefit of maintenance therapy following initial BCG induction (7). Meta-analyses reported that BCG maintenance significantly reduces recurrence or progression in patients with NMIBC $(8,9)$. Several studies, however, revealed no benefit of maintenance therapy in controlling tumor recurrence or progression (10-12). Differences in the results are possibly due to the maintenance schedules. The maintenance schedules varied considerably and it appears that a weekly, monthly, quarterly or 6-monthly schedule for 1-2 years may be immunologically suboptimal $(4,10,11,13)$. The aim of this study was to confirm the recurrence- and progression-preventing efficacy and safety of 12 times monthly BCG maintenance therapy for NMIBC.

\section{Materials and methods}

This case-control study included 126 patients diagnosed with Ta, T1 and CIS bladder cancer between January 2000 and 
December 2009, who received intravesical immunotherapy. The inclusion criteria were high-risk NMIBC and recurrent NMIBC, such as, pathologically confirmed Ta, T1 and CIS of the bladder, administration of intravesical initiation or maintenance immunotherapy, performance status of $0-2$, ability to undergo cystoscopy and TUR-BT, and intact function of main organs (14). Exclusion criteria included reduced dosage of BCG Tice strain, failure of consecutive treatment, strongly positive tuberculin test, history of intravesical BCG instillation, severe bladder irritation before the start of BCG administration and active serious medical complications. Of 126 patients with NMIBC, 34 patients in the no maintenance group received a single 6-week course of intravesical immunotherapy after TUR-BT. Ninety-two patients in the maintenance group received 12 times monthly maintenance therapy course after a single 6-week course of intravesical immunotherapy after TUR-BT.

Prior to intravesical immunotherapy, patients provided a health history and underwent a physical examination, urinalysis, urine cytology, complete blood count, blood urea nitrogen, serum creatinine, liver function test and chest $\mathrm{X}$-ray. The single 6-week course of intravesical immunotherapy started within 1 week after TUR-BT with $12.5 \mathrm{mg}$ of BCG Tice strain (OncoTICE; Oregano, West Orange, NJ, USA). BCG Tice strain was first suspended in $3 \mathrm{ml}$ diluents, followed by dilution with $40 \mathrm{ml}$ physiological saline. That suspension was then instilled into the bladder via a urethral catheter. Patients were required to hold their urine for $\sim 2 \mathrm{~h}$ after instillation (15). BCG treatment was repeated weekly for 6 consecutive weeks. An additional 12 times monthly course after the single 6-week course of intravesical immunotherapy was initiated 1 month later after a single 6-week course with BCG Tice strain. BCG treatment was repeated monthly for 12 consecutive months.

Efficacy of the treatment regimens was assessed on the basis of cystoscopy and urinary cytology finding. The patients were followed up every 3 months during the first 2 years, and every 6 months thereafter (14). End-points were RFS and progression-free survival (PFS). When tumor recurrence was suspected on the basis of the findings of cystoscopy and urinary cytology, confirmation was carried out by performing TUR-BT. Progression at the time of recurrence was defined as pathological stage T2 disease or greater, or the use of cystectomy, systemic chemotherapy or radiation therapy. Kaplan-Meier survival curves were used to estimate disease-free survival. P-value $<0.05$ was considered to denote statistical significance.

\section{Results}

The median follow-up period was 43 months (interquartile range 18-64; range 3-123) in the maintenance group and 16.5 months (interquartile range 6-35.75; range 3-75) in the no maintenance group. The baseline clinicopathological characteristics of the NMIBC patients are shown in Table I. One hundred and twenty-six patients were eligible for enrollment in our study. In total, 92 patients with $\mathrm{Ta}(\mathrm{n}=25)$ and $\mathrm{T} 1(\mathrm{n}=67)$ were treated with intravesical BCG maintenance therapy, and 34 patients with Ta $(n=15)$ and $T 1(n=19)$ were treated with intravesical BCG initiation therapy. There were no significant
Table I. Baseline characteristics of the maintenance and no maintenance groups.

\begin{tabular}{|c|c|c|c|}
\hline Characteristics & $\begin{array}{c}\text { Maintenance } \\
(\mathrm{n}=92)\end{array}$ & $\begin{array}{l}\text { No maintenance } \\
\quad(\mathrm{n}=34)\end{array}$ & P-value \\
\hline Gender & & & 0.273 \\
\hline Male & 75 & 31 & \\
\hline Female & 17 & 3 & \\
\hline Age (years) & $61.73 \pm 11.865$ & $65.88 \pm 11.762$ & 0.083 \\
\hline T stage & & & 0.086 \\
\hline $\mathrm{Ta}$ & 25 & 15 & \\
\hline $\mathrm{T} 1$ & 67 & 19 & \\
\hline Carcinoma in situ & & & 0.526 \\
\hline Yes & 9 & 5 & \\
\hline No & 83 & 29 & \\
\hline Grade & & & 0.520 \\
\hline G1 & 14 & 6 & \\
\hline G2 & 47 & 14 & \\
\hline G3 & 28 & 14 & \\
\hline Multiplicity & & & 0.677 \\
\hline Single & 33 & 11 & \\
\hline Multiple & 55 & 23 & \\
\hline Recurrence history & & & 0.793 \\
\hline Primary & 75 & 27 & \\
\hline Recurrent & 15 & 7 & \\
\hline Smoking history & & & 0.178 \\
\hline Yes & 29 & 23 & \\
\hline No & 25 & 10 & \\
\hline
\end{tabular}

Table II. Toxicity and side effects of the maintenance and no maintenance groups.

\begin{tabular}{|c|c|c|}
\hline Characteristics & $\begin{array}{l}\text { Maintenance } \\
\quad(\mathrm{n}=92)\end{array}$ & $\begin{array}{l}\text { No maintenance } \\
\quad(n=34)\end{array}$ \\
\hline Overall side effects & $37(40.2)$ & $15(44.1)$ \\
\hline \multicolumn{3}{|l|}{ Local toxicity } \\
\hline Irritative symptom & $30(32.6)$ & $14(41.1)$ \\
\hline Low-grade fever & $1(1.1)$ & $0(0.0)$ \\
\hline Gross hematuria & $5(5.4)$ & $2(5.8)$ \\
\hline Tuberculosis cystitis & $2(2.2)$ & $0(0.0)$ \\
\hline Tuberculosis prostatitis & $1(1.1)$ & $0(0.0)$ \\
\hline \multicolumn{3}{|l|}{ General toxicity } \\
\hline High-grade fever & $2(2.2)$ & $0(0.0)$ \\
\hline Pulmonary tuberculosis & $1(1.1)$ & $0(0.0)$ \\
\hline
\end{tabular}

clinicopathological differences between the maintenance and no maintenance groups.

The complications and side effects of immunotherapy are reported in Table II. The incidence of overall side effects was $40.2 \%$ in the maintenance group and $44.1 \%$ in the no maintenance group. Most of these complications were irritative symptoms (frequency, urgency and tenesmus). Two patients who had high-grade fever during maintenance therapy were 


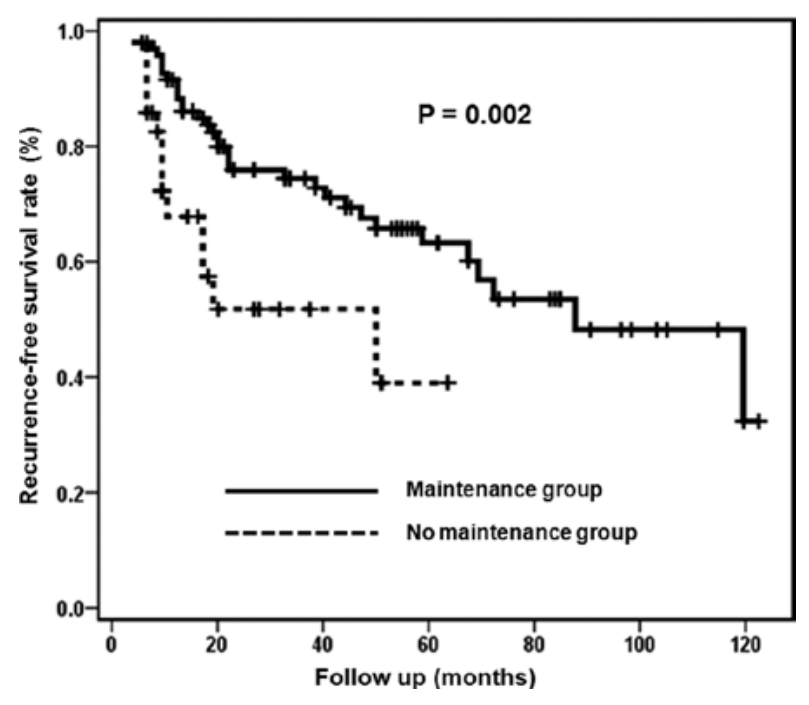

Figure 1. Recurrence-free survival curves of the maintenance and no maintenance groups.

treated with antipyretic medication. However, these patients had finished maintenance therapy. One patient who finished maintenance therapy had pulmonary tuberculosis.

Fig. 1 shows RFS curves of the maintenance and no maintenance groups. The estimated median RFS was 87 months (95\% CI 53.0-120.9) in the maintenance group and 48 months (95\% CI 0-96.8) in the no maintenance group (log-rank test; $\mathrm{P}=0.002$ ). The 2-year cumulative RFS rates were $77.3 \%$ in the maintenance group and $52.6 \%$ in the no maintenance group. The 5-year cumulative RFS rates were $64.4 \%$ in the maintenance group and $39.4 \%$ in the no maintenance group.

Fig. 2 shows PFS curves of the maintenance and no maintenance groups. Median PFS rates were not estimable in both groups (log-rank test; $\mathrm{P}=0.178$ ). The 2-year cumulative PFS rates were $91.1 \%$ in the maintenance group and $80.5 \%$ in the no maintenance group. The 5 -year cumulative PFS rates were $80.0 \%$ in the maintenance group and $71.6 \%$ in the no maintenance group.

Fig. 3 shows disease-specific survival (DSS) curves of the maintenance and no maintenance groups. Median DSS rates were not estimable in both groups (log-rank test; $\mathrm{P}=0.111$ ). The 2-year cumulative DSS rates were $97.7 \%$ in the maintenance group and $91.4 \%$ in the no maintenance group. The 5-year cumulative DSS rates were $85.3 \%$ in the maintenance group and $74.3 \%$ in the no maintenance group.

\section{Discussion}

Morales et al reported that adjuvant intravesical BCG therapy reduced tumor recurrence (3). Large randomized clinical trials performed by the SWOG demonstrated that BCG immunotherapy provides significantly longer time to first recurrence compared to chemotherapy. A randomized SWOG study reported that estimated median RFS was 76.8 months (95\% CI 64.3-93.2) in the maintenance arm and 35.7 months (95\% CI 25.1-56.8) in the no maintenance. They concluded that the overall 5-year survival was $83 \%$ in the maintenance arm compared to $78 \%$ in the no maintenance arm (4). Our study, using a monthly schedule, produced similar results: the esti-

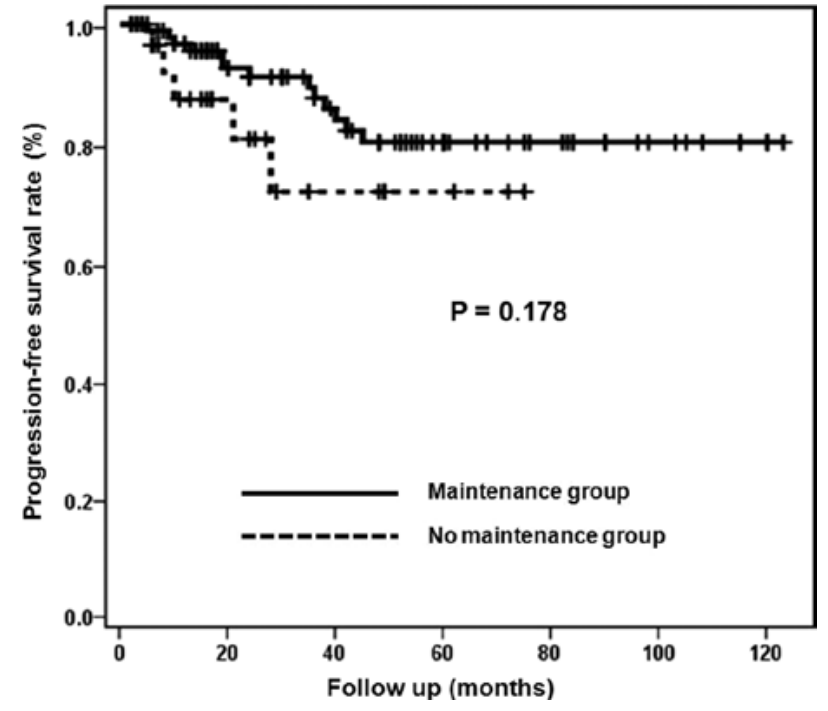

Figure 2. Progression-free survival curves of the maintenance and no maintenance groups.

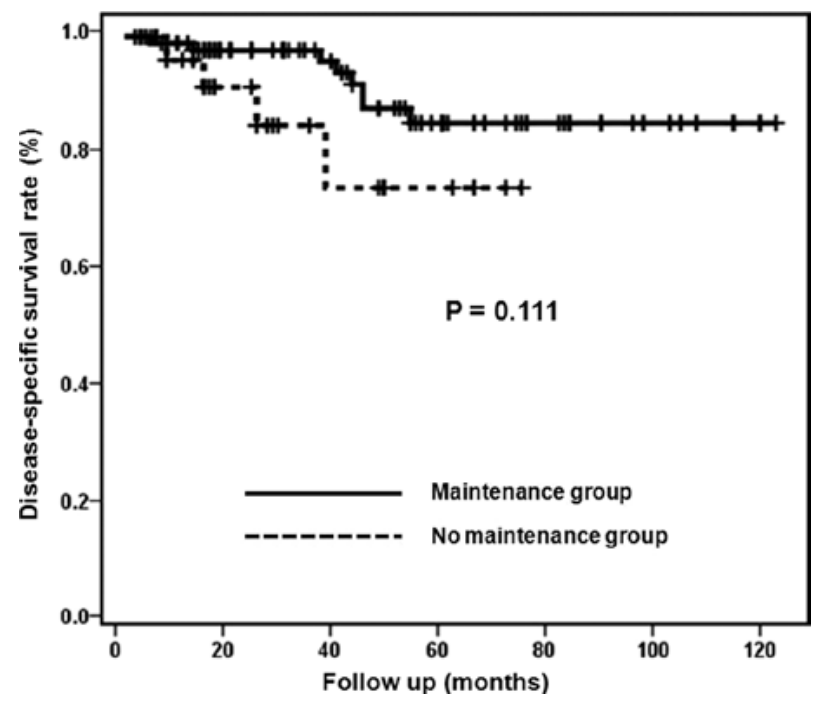

Figure 3. Disease-specific survival curves of the maintenance and no maintenance groups.

mated median RFS was 87 months (95\% CI 53.0-120.9) in the maintenance group and 48 months (95\% CI 0-96.8) in the no maintenance group. The 5 -year survival rates were $85.3 \%$ in the maintenance group and $74.3 \%$ in the no maintenance group.

Two meta-analyses showed that intravesical BCG maintenance therapy reduced the risk of tumor progression $(8,9)$. Sylvester et al reported that intravesical BCG significantly reduced the risk of tumor progression after transurethral resection in patients with superficial bladder cancer who received maintenance treatment. They identified 24 trials with progression information on 4,863 patients, and 260 of 2,658 patients in the BCG maintenance group (9.8\%) had progression compared to 304 of 2,205 patients in the no maintenance group (13.8\%) (8). Bohle et al revealed similar results for BCG prevention of tumor progression. They reported that BCG maintenance showed a statistically significant superiority over mitomycin C $(\mathrm{OR}=0.66 ; 95 \%$ CI $0.47-0.94)(9)$. 
Several studies, however, have not been able to show that intravesical chemotherapy and immunotherapy have an influence on the time to progression to muscle invasive disease $(4,16,17)$. Lamm et al found that maintenance chemotherapy failed to improve significant benefit of early post-operative intravesical chemotherapy. These data showed no decreased tumor progression, and overall among 2,011 randomized patients progression occurred in $7.5 \%$ of those receiving intravesical chemotherapy and $6.9 \%$ of those treated by surgery alone (17). Pawinski et al reported that no clear advantage of adjuvant treatment was shown with respect to progression to invasion, time to appearance of distant metastases or duration of survival and PFS. They concluded that, despite prolongation of the disease-free interval, adjuvant treatment has no apparent long-term impact on the evolution of stage Ta and T1 bladder cancer (16). The SWOG study reported that the estimated median time for worsening-free survival, defined as no evidence of progression, including pathological stage T2 disease or greater, or the use of cystectomy, systemic chemotherapy or radiation therapy, was 111.5 months in the no maintenance and not estimable in the maintenance arm (4).

Two monthly maintenance therapies for NMIBC have been reported $(10,18)$. In a study by Badalament et al, 46 patients received BCG weekly for 6 weeks and were compared to 47 patients receiving the 6-weekly doses of BCG plus monthly BCG for 2 years. They concluded that patients receiving maintenance and no maintenance therapy had similar tumor recurrence and progression rates. These results indicate that monthly maintenance BCG does not prevent, delay or reduce tumor recurrence or progression observed with the 6-week regimen (10). In a study by Akaza et al, 39 patients receiving prophylactic maintenance therapy consisting of BCG of $40 \mathrm{mg}$ monthly for 12 times were compared to 55 patients not receiving maintenance therapy. After a 3-year follow-up period, the final cumulative RFS rates were $77.6 \%$ in the maintenance group and $74.2 \%$ in the no maintenance group. They concluded that transurethral resection of tumors of the bladder alone is unlikely to eliminate the bladder tumor, and intravesical BCG is potentially the treatment of choice.

Our previous study examined changes in bladder mucosal immune cells in patients with superficial bladder carcinoma treated with BCG and doxorubicin. The post-treatment bladder mucosal B cells and $\mathrm{T}$ cells were significantly increased compared to pre-treatment in patients treated with BCG instillation. There was, however, no change in B or T cells in patients treated with doxorubicin. There was no tumor recurrence in cases with significantly increased numbers of B cells after BCG instillation. The results of our study suggest that intravesical BCG immunotherapy induces a significant increase in T cells as well as B cells, and B cells have a preventive effect on tumor recurrence. We conclude that stimulation at regular intervals may be effective to activate $\mathrm{T}$ and $\mathrm{B}$ cells, such as booster injection for immunization (19).

Regarding tumor progression, our results showed that the 2-year cumulative PFS rates were $91.1 \%$ in the maintenance group and $80.5 \%$ in the no maintenance group. However, the difference was not statistically significant. Maintenance therapy of the SWOG study consisted of intravesical and percutaneous BCG each week for 3 weeks, administered 3,6,
$12,18,24,30$ and 36 months from the initiation of induction therapy (4). The total number of maintenance therapy sessions was 21 in the SWOG study and 12 in our study. This difference may have affected the PFS rates. In addition, the difference in the number of patients between the maintenance and no maintenance groups and the small sample size reduced the statistical significance of the results. These were limitations of our case-control study.

\section{Acknowledgements}

This study was supported by the Korea Science and Engineering Grant Foundation of the Korean Government (no. 20100028333).

\section{References}

1. Jemal A, Bray F, Center MM, Ferlay J, Ward E and Forman D: Global cancer statistics. CA Cancer J Clin 61: 69-90, 2011.

2. Aldousari $\mathrm{S}$ and Kassouf W: Update on the management of nonmuscle invasive bladder cancer. Can Urol Assoc J 4: 56-64, 2010.

3. Morales A, Eidinger D and Bruce AW: Intracavitary bacillus Calmette-Guerin in the treatment of superficial bladder tumors. J Urol 116: 180-183, 1976.

4. Lamm DL, Blumenstein BA, Crissman JD, et al: Maintenance bacillus Calmette-Guerin immunotherapy for recurrent TA, T1 and carcinoma in situ transitional cell carcinoma of the bladder: a randomized Southwest Oncology Group Study. J Urol 163: 1124-1129, 2000.

5. Herr HW, Wartinger DD, Fair WR and Oettgen HF: Bacillus Calmette-Guerin therapy for superficial bladder cancer: a 10-year follow-up. J Urol 147: 1020-1023, 1992.

6. Pansadoro V, Emiliozzi P, Defidio L, et al: Bacillus CalmetteGuerin in the treatment of stage T1 grade 3 transitional cell carcinoma of the bladder: long-term results. J Urol 154: 2054-2058, 1995.

7. Shelley MD, Mason MD and Kynaston H: Intravesical therapy for superficial bladder cancer: a systematic review of randomised trials and meta-analyses. Cancer Treat Rev 36: 195-205, 2010.

8. Sylvester RJ, van der MA and Lamm DL: Intravesical bacillus Calmette-Guerin reduces the risk of progression in patients with superficial bladder cancer: a meta-analysis of the published results of randomized clinical trials. J Urol 168: 1964-1970, 2002.

9. Bohle A and Bock PR: Intravesical bacille Calmette-Guerin versus mitomycin $\mathrm{C}$ in superficial bladder cancer: formal meta-analysis of comparative studies on tumor progression. Urology 63: 682-687, 2004.

10. Badalament RA, Herr HW, Wong GY, et al: A prospective randomized trial of maintenance versus nonmaintenance intravesical bacillus Calmette-Guerin therapy of superficial bladder cancer. J Clin Oncol 5: 441-449, 1987.

11. Hudson MA, Ratliff TL, Gillen DP, Haaff EO, Dresner SM and Catalona WJ: Single course versus maintenance bacillus Calmette-Guerin therapy for superficial bladder tumors: a prospective, randomized trial. J Urol 138: 295-298, 1987.

12. Akaza H, Kameyama S, Kakizoe T, et al: Ablative and prophylactic effects of BCG Tokyo 172 strain for intravesical treatment in patients with superficial bladder cancer and carcinoma in situ of the bladder. Bladder cancer BCG Study Group. Nippon Hinyokika Gakkai Zasshi 83: 183-189, 1992 (In Japanese).

13. Palou J, Laguna P, Millan-Rodriguez F, Hall RR, Salvador-Bayarri J and Vicente-Rodriguez J: Control group and maintenance treatment with bacillus Calmette-Guerin for carcinoma in situ and/or high grade bladder tumors. J Urol 165: 1488-1491, 2001

14. Koga H, Ozono S, Tsushima T, et al: Maintenance intravesical bacillus Calmette-Guerin instillation for Ta, T1 cancer and carcinoma in situ of the bladder: randomized controlled trial by the BCG Tokyo Strain Study Group. Int J Urol 17: 759-766, 2010

15. Jung S, Jung SI and Chung JI: Bacillus Calmette-Guerin intravesical therapy in superficial bladder cancer: the early experience of comparison of 6 week course and modified 63 maintenance therapy. Korean J Urol 49: 703-708, 2008. 
16. Pawinski A, Sylvester R, Kurth KH, et al: A combined analysis of European Organization for Research and Treatment of Cancer, and Medical Research Council randomized clinical trials for the prophylactic treatment of stage TaT1 bladder cancer. European Organization for Research and Treatment of Cancer Genitourinary Tract Cancer Cooperative Group and the Medical Research Council Working Party on Superficial Bladder Cancer. J Urol 156: 1934-1941, 1996.

17. Lamm DL, Riggs DR, Traynelis CL and Nseyo UO: Apparent failure of current intravesical chemotherapy prophylaxis to influence the long-term course of superficial transitional cell carcinoma of the bladder. J Urol 153: 1444-1450, 1995.
18. Akaza H, Hinotsu S, Aso Y, Kakizoe T and Koiso K: Bacillus Calmette-Guerin treatment of existing papillary bladder cancer and carcinoma in situ of the bladder. Four-year results. The Bladder Cancer BCG Study Group. Cancer 75: 552-559, 1995.

19. Chang SG, Lee SJ, Huh JS and Lee JH: Changes in mucosal immune cells of bladder tumor patient after BCG intravesical immunotherapy. Oncol Rep 8: 257-261, 2001. 